\title{
Data Visualization of the Advertising Exchange Flow Statements Generation System on Demand Side Platform Based on Highcharts
}

\author{
Jin Bao Song ${ }^{1, a}$, Jin Hong Song ${ }^{2}$, Jian Ping Chai ${ }^{3}$ and Yue Yang ${ }^{4}$ \\ ${ }^{1}$ Information Engineering School, Communication University of China, Beijing, China \\ ${ }^{2}$ Shandong Gold Mining Jiaojia Gold Mine (Laizhou) Co., Ltd, Shandong, China \\ ${ }^{3}$ Faculty of Science and Techonology, Communication University of China, Beijing, China \\ ${ }^{4}$ Information Engineering School, Communication University of China, Beijing, China
}

\begin{abstract}
Research shows that the information human obtained about the external world are more than $80 \%$ by visual access channel, especially in the background of today's era of the big data. The value of data visualization is particularly important. The flow statements generation system for advertising exchange on Demand-Side Platform based on Real Time Bidding is mainly to solve the large volume of data of flow observation statements and the inefficiency of artificial sorting. It sets RTX Single Sign On and Reverse Login to certificate user's identity through JAVA development, screening flow data according to the platform and region, and invoke Highcharts to generate advertisement exchange flow observation report automatically. In this paper, the data visualization and charts showing are regarded as the final goal, through the introduction of the Spring MVC framework, Eclipse development environment, Highcharts charts generation, JAVA programming language, MySQL database and some other related knowledge. It is to show readers the whole development ideas clearly of the flow statements generation system for advertising exchange on Demand-Side Platform, at the same time the visualized charts by running the whole program codes will be shown.
\end{abstract}

Keywords: Data Visualization, Flow Statements of Advertising Exchange, Java, Highcharts.

\section{Introduction}

The flow statements generation system for advertising exchange based on RTB is to develop a practical Internet interactive system to solve the complex and low efficiency problems of manual sorting data flow statements. The secondary development is done based on Tencent office software RTX in order to increase the function of the single sign-on (SSO) and reverse login and to make the office automation a great extent display. At the same time the database is connected, the classified and filtered advertising exchange flow data is shown on the Web page in the form of lists and charts. The function of the chart print and preservation is realized. The advertisers can monitor the change of advertising exchange flow more clearly and conveniently [1].

Real Time Bidding (RTB) is a kind of bidding technology to evaluate and bid for each user showing behavior on hundreds of millions of websites using third-party technology. Traditional Internet advertising ecosystem usually only has up to three sides including advertisers, advertising agents and the Internet media. However, in Real Time Bidding (RTB) advertising exchange mode, the whole advertising chain has four main bodies including advertisers, the advertising
Demand Side Platform (DSP), advertising exchange Platform (Ad Exchange) and the Internet media. Advertisers put own advertising demand on DSP platform. The Internet media puts own advertising flow resources into the advertising exchange platform. DSP completes bidding to buy through docking with the advertising exchange platform technology. When the users access a web site, the Sell Side Platform (SSP) sends the users access signal to advertising exchange platform. Then the detail advertising information will be sent to DSP through the analysis matching of Decision Making Process (DMP). DSP will be bidding. The advertising position will be obtained by the highest price provider. The corresponding advertisement will be shown to target users. The advertising delivery will finish. The advertising real time bidding process flow chart is as shown in Fig. 1. 


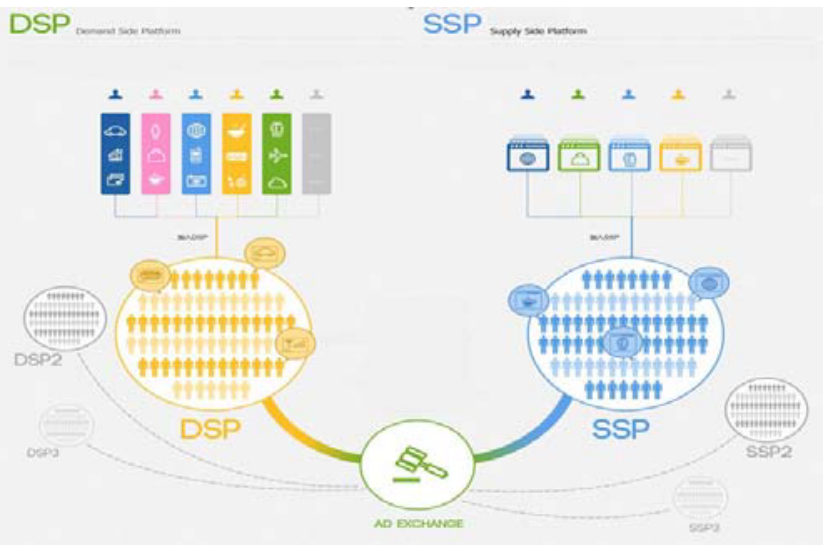

Figure 1. The advertising real time bidding process flow chart

There is the new change in the Internet advertising industry since 2011. Real time bidding (RTB) technology appears in the field of display advertising. The traditional artificial negotiation and flow package pattern slowly are reversed. The previous media advertising has been unable to meet the more and more refined requirements of advertisers. By the end of 2012, the domestic RTB advertising proportion in the whole display advertising is still very small. Today, more than 40 domestic online advertising DSPs are born, such as Easy Media, IPINYOU, etc. On the media side, Tencent, Baidu, Alibaba launch advertising exchange platform. DSP services for the advertisers or agency, in the form of RTB, purchases a variety of media resources (including Ad Network, SSP, Ad Exchange, etc). It is believed that real time bidding (RTB) advertising patterns will be roundly transplanted to advertising market in China soon.

In order to allow advertisers to understand the practical effects of the advertising, the demand side platform (DSP) need for real-time monitoring of advertising exchange flow. The data flow information obtained from advertising exchange flow monitoring tool is stored in DSP internal database. It is necessary for advertisers and DSP internal operating personnel to check and monitor advertising data flow regularly. By checking the flow percentage chart, click rate and the click unit price comparison chart, and participate rate and success rate in the bidding comparison chart of each platform, the development stage of advertising exchange flow can be seen clearly and the corresponding measures can be put forward in time. The required data for chart show was artificially and manually selected from the database ever before. Then a chart is made to view by using Excel. However, the rapid development of real time bidding business also has increasing advertising exchange flow observation data. And it is more and more difficult for flow monitoring and data analysis. The manual sorting flow data reports is very big workload. There are the shortcomings of low efficiency and time-consuming. Thus it becomes a pressing problem that DSP can realize the automatic generation of advertising exchange flow observation report.
Aiming at the three mainstream advertising exchange platform, this paper mainly researches the pie chart of flow occupancy rate for different platform generated by using the Highcharts, participate rate and success rate in the bidding each platform, and the comparison chart of the advertising effect and the flow utilization of each platform. The data visualization of related aspects is also researched [2].

The flow statements generation system for advertising exchange based on RTB mainly regards the data chart show as the core. It is the secondary development to office automation (OA) system RTX in order to increase the function of the single sign-on (SSO) and reverse login. Then the database is connected. The advertising exchange flow data is classified and filtered. The chart show is achieved together by invoking the Highcharts. The problem of low efficiency of manual sorting and complexity of advertising exchange flow data is resolved. The shown chart is not only beautiful and easy but also can realize data visualization. It makes the enterprise internal operational staff more convenient to analysis and sort data at the same time. The rise and decline of advertising exchange flow data and effectiveness of the market can be seen more intuitively.

\section{Spring MVC}

The flow data filtering and statements show include two parts of data statement and data graph display. The two parts are completed in the Spring MVC framework. The statement display is connected to MySQL database. The required data tables are queried for the advertisers or development operation personnel. Through the JAVA programming, the required data is displayed on the JSP page in the form of table. And data graph display part is mainly developed by using JAVA language and calling a graph library Highcharts written by pure JavaScript. Highcharts is simple and convenient to add interactive charts in the Web site or Web application. Highcharts interface is aesthetic. Due to the use of JavaScript code, so Highcharts don't need plug-in to run like Flash and JAVA. And Highcharts runs fast. HighCharts had a good compatibility. It will be able to support most of the current browsers. In the development process, by connecting to databases, the flow data can be filtered for each platform and can be made into needed chart data items through the operation. Then data can be converted to the JSON data format. The data can be gotten by using AJAX. The pie chart, rectangle and line - rectangular mixed chart are automatically generated by Highcharts [3].

Spring is a layered Java SE/EE application one-stop lightweight open source framework [4]. Spring's biggest feature is as a kind of container providing class. Spring is one of the factory pattern evolution version. The core in Spring is the BeanFactory. The object instantiation of the programmer is concentrated in the Spring configuration file [5]. On the suggestion of adopting programming to an interface, the highly coupling between the programs is realized by Spring. Two key 
technologies of Spring are inverse of control (IOC) and aspect oriented programming (AOP) [6].

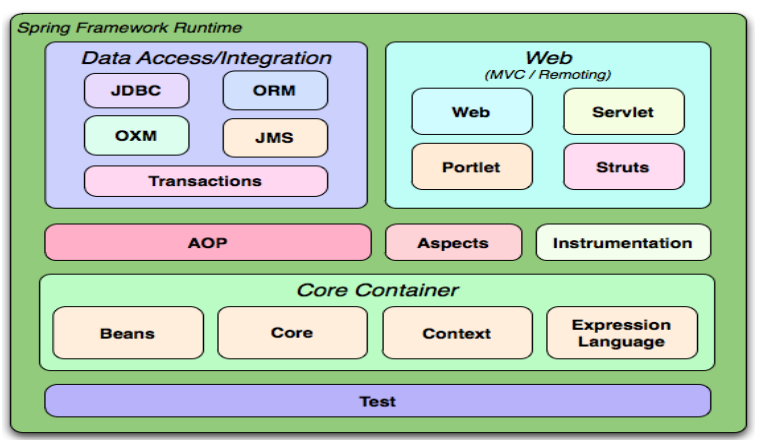

Figure 2. Spring schematic framework

\section{Module Demand Analysis and Function Design}

The Internet advertising exchange industry is continuously flourishing today. This new exchange form of the advertising real time bidding gradually win the favor of many advertisers and advertising exchange platform. More and more advertisers choose to place their own advertising demand on the demand side platform (DSP). At the same time, the Internet media advertising and other media resources will be provided on demand side platform (DSP). When the user browses the mainstream web site which has established cooperation with the demand side platform, the Sell Side Platform (SSP) sends the users access signal to advertising exchange platform. The advertising exchange platform launch real time bidding for different size of advertising position. The highest bidding price is success. Advertising can be placed. The whole process of advertising exchange is not only fast but also can advertise according to the crowd orientation. It has particularly good effects. But it also can produce a new problem at the same time. The increasing advertising exchange flow makes the advertisers become very complex for flow monitoring. So the demand side platform will need to build a platform that can automatically call flow data in the database and automatically generate flow analysis chart, which is advantageous for the advertisers to access and monitor.

The flow statements generation system for advertising exchange based on RTB includes three modules: the module of user register and login, the module of statement display and the module of chart display. The module of chart display includes three charts: the flow percentage chart of each platform as shown in Fig. 3, click rate and the click unit price comparison chart of each platform as shown in Fig. 4, and participate rate and success rate in the bidding comparison chart of each platform as shown in Fig. 5.

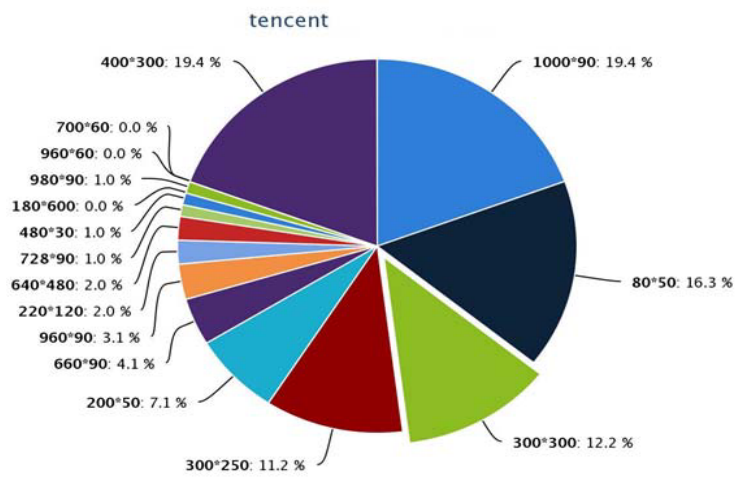

Figure 3. The flow percentage chart of each platform

$\tan x$

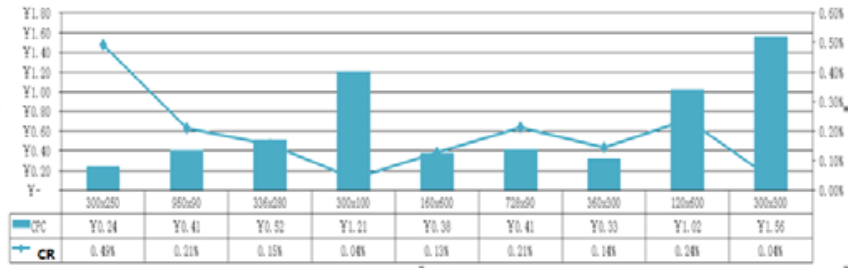

Figure 4. Click rate and the click unit price comparison chart of each platform

$A D X$

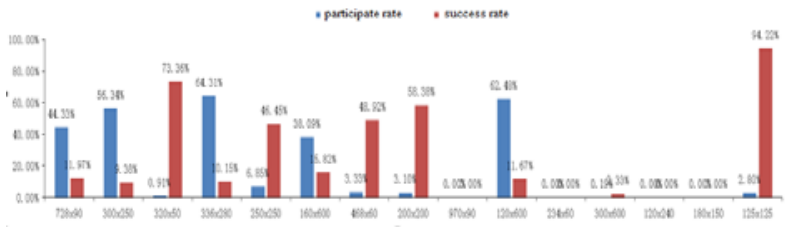

Figure 5. Participate rate and success rate in the bidding comparison chart of each platform

\section{Summary}

The flow statements generation system for advertising exchange based on RTB is mainly completed in the Spring MVC framework, using the Eclipse development platform, the MySQL database knowledge, the JAVA language development technology and JSP technology, etc. Research shows that the information human obtained about the external world are more than $80 \%$ by visual access channel, especially in the background of today's era of the big data. The value of data visualization is particularly important. The flow statements generation system for advertising exchange on Demand-Side Platform based on Real Time Bidding is mainly to solve the large volume of data of flow observation statements and the inefficiency of artificial sorting. It sets RTX Single Sign On and Reverse Login to certificate user's identity through JAVA development, screening flow data according to the platform and region, and invoke Highcharts to generate advertisement exchange flow observation report automatically. The flow statements generation system for advertising exchange based on RTB solves the problem of low efficiency of manual sorting and complexity of advertising exchange flow data and has good practical applications. The data 
visualization based on Highcharts also has a broad development prospect.

\section{References}

[1] Z. B. Liu, Java WEB core framework. Beijing: Posts \& Telecom Press, 2013, pp. 123-135.

[2] R. Johnson and J. Hoeller, Expert one-on-one J2EE Development without EJB, 2015.

[3] J. Kuan, Learning Highcharts, Packt Publishing Limited, 2013.

[4] Johnson R and Hoeller J,The Spring Framework Reference Documentation, 2012, http: / / static. springframework.org/ spring/ docs/ 2. 5. x/ reference/.

[5] Alur D, Crupi J and Malks D, Core J2EE Patterns: Best Practices and Design Strategies, American: Prentice Hall, 2013.

[6] M. Fowler, Patterns of Enterprise Application Architecture, 2011. 